\title{
$\mathrm{N} 92-21483$
}

\section{VISUAL DIRECTION AS A METRIC OF VIRTUAL SPACE}

\author{
Stephen R. Ellis, ${ }^{1}$ Stephen Smith, ${ }^{2}$ Selim Hacisalihzade ${ }^{3}$ \\ NASA Ames Research Center \\ Moffett Field, California
}

\begin{abstract}
Two experiments examine the abilities of 10 subjects to visualize directions shown on a perspective display. Subjects indicated their perceived directions by adjusting a head-mounted cursor to correspond to the direction depicted on the display. This task is required of telerobotic operators who use map-like pictures of their workspace to determine the direction of objects seen by direct view. Results show significant open-loop, judgement biases that may be composed of errors arising from misinterpretation of the map geometry and overestimation of gaze direction.
\end{abstract}

\section{INTRODUCTION}

A number of investigations and reviews of the characteristics of the virtual space perceived in pictures have been conducted recently (Rosinski et al.,1980; Sedgwick,1986; McGreevy and Ellis, 1986; Grunwald and Ellis, 1986; Ellis, Smith and McGreevy, 1987; Barfield, Sandford, and Foley, 1989). Despite the fact that the pictures considered were not stereoscopic, viewers typically were reported to develop a clear sense that the pictured objects were laid out in a virtual space. Quantitative characterization of the metrics of the viewer's perceived space will advance our understanding of picture perception and assist the design of displays for aircraft and spacecraft. The objective of the following research is to characterize patterns of errors observers make when referring a judged exocentric direction to a target presented on a perspective display to their own egocentric sense of visual direction. This type of spatial task is commonly faced by operators of telerobotic systems when using a map-like display of their workspace to determine the visual location and orientation of objects seen by direct view. It is also essentially the same task as faced by an aircraft pilot using a cockpit perspective traffic display of his surrounding airspace to locate traffic out his windows.

Previous studies of the error pattern in direction judgements have focused on exocentric judgements for which the subjects indicated their estimates of the target position by adjusting dials to show a target's azimuth and elevation with respect to a reference direction vector (See fig. 1).

\footnotetext{
${ }^{1}$ NASA Ames Research Center and U.C. Berkeley School of Optometry

${ }^{2}$ Sterling Software, Palo Alto, CA

${ }^{3}$ NRC Research Associate, NASA Ames Research Center
} 
This response may be described as exocentric since the dial's frame of reference is external to the observer and contrasts with egocentric judgements in which target position is indicated with respect to a body-referenced coordinate system Accordingly, in order to test the generality of reported biases in estimating azimuth and elevation with exocentric judgements, it is useful to examine the same exocentric task but request the subjects to make egocentric judgements.

For this new response the observer adjusts the visual direction of head-mounted light cursor to indicate his sense of the target's depicted azimuth and elevation with respect to a reference position and reference direction. This response will explicitly test the generality of previously reported bias in which exocentric directions are judged to be away from a reference straight ahead. This bias may be attributed to errors in the subjects ability to determine the view direction used to generate the display (McGreevy and Ellis, 1986; Grunwald and Ellis, 1986; Ellis, Smith, Grunwald, and McGreevy, 1989). Furthermore, use of an egocentric response such as visual direction provides a more natural response than a dial adjustment. In a sense we ask the subjects to imagine themselves oriented in the virtual space along a particular direction vector and then to imagine where they would have to look to see the target.

\section{METHODS}

Two groups of 5 subjects participated as independent groups in two experiments. The subjects were male laboratory personnel ranging in age from 20 to 43 who were unfamiliar with the purpose of the experiment.

The experiments were conducted inside a $1.5 \mathrm{~m}$ planetarium dome that served as a projection surface for a head mounted, light pointer which projected a red filament image shaped as a $1.5^{\circ}$ chevron onto the dome's surface (light from a $3 \mathrm{v}$ flashlight bulb through a Wratten \#25 filter). The subject's head position was sensed by a Polhemus electromagnetic head tracker attached to an nonmetallic modified welder's helmet approximately $11 \mathrm{~cm}$ above the head. The head tracker was independently calibrated against 28 theodolite-positioned, reference markers which were visible during calibration but not during testing.

The subjects were presented with an exocentric judgement task generated by a PDP 11/40 Evans \& Sutherland PS I graphics system. The images used were similar to earlier experiments (McGreevy and Ellis, 1986; Grunwald and Ellis, 1986; Ellis, Smith, Grunwald, and McGreevy, 1989). The major change was the greater yaw of the view direction used to create the images. It was set to a counterclockwise yaw of $-35^{\circ}$. Pitch remained $-22^{\circ}$. The subjects were seated at the center of the projection in front of the computer calligraphic monitor about $80 \mathrm{~cm}$ from the display surface and looked downward into it with a $-22 \mathrm{deg}$. pitch angle matching that of the view vector. The viewport was $17 \mathrm{~cm}$ square.

Subjects were first positioned in an adjustable chair so that their head-mounted light cursor pointed to a subjective straight-ahead, eye level that corresponded to the calibration point at $0^{\circ}$ pitch and $0^{\circ}$ yaw. (See fig. 2) While in this position, a reference reading was taken from the head sensor

for all future measurements. The subjects then were instructed to examine a series of automatically, 
randomly presented displays and to estimate the azimuth and elevation direction of the target with respect to a reference position and direction. Then they were to transfer this judgement to their egocentric frame of reference. They made the judgement by adjusting the pitch and yaw of their headmounted, light cursor to a position where they would expect to see the target if their head was at the reference position, and initially aligned with the reference direction in the displayed virtual space. For most of the judged directions the subjects could not simultaneously see the display and the cursor position, but had to gaze back and forth between them to accomplish the task, generally using head movements for excentricites greater than $15^{\circ}$. After adjusting the cursor, they held their position and moved a toggle switch that signaled the computer to take the data. The data for a $1 \mathrm{sec}$. period prior to the switch signal were averaged to give a single measurement. Three replications of each position were taken from each subject in a randomized sequence of 64 measurements that took about 2 hours to complete.

The interpretation of the head-direction data is complicated by the different centers of rotation associated with pitch, yaw and roll of the head. Pure yaws did not displace the center of rotation very much and the measured head yaw to the calibrated positions were within $2^{\circ}$ of the calibrated angles within $\pm 60^{\circ}$ of the straight ahead, the greatest deviations being at the most extreme angles. The reason for the residual error was the difficulty of exactly positioning the subject to the calibration reference point. Pitch in contrast tends to be around a moving center of rotation somewhat behind the neck and consequently tends to translate the head upwards and backwards from the initial reference point which was used to provide a straight ahead, level reference for all subsequent measures. Consequently, when the subjects pointed their head-mounted cursors to the extreme pitches, the sensor reading undershot the calibrated value by from 5 to 8 degrees! We have calculated geometrical corrections for the effects of this displacement from the reference point since we could measure it, but generally found that they were small $\left(2-4^{\circ}\right)$ and for reasons discussed below may not in principle be proper to use.

After calibration of the head tracker in the light, the two experiments were conducted in the dark with the CRT display turned down so that only the frame of the monitor was faintly visible to provide an egocentric direction reference. In one experiment the head cursor was kept on. In the other the cursor was turned off and the subjects had to rely principally on vestibular and proprioceptive cues to "look" to the direction they would expect to see the target.

\section{RESULTS AND DISCUSSION}

The results from both experiments were similar and are analyzed together in this summary. Multivariate analysis of variance conducted with BMDP $4 \mathrm{~V}$ on the elevation and azimuth errors showed that for both judgements the target elevation, target azimuth had statistically reliable effects on both the pitch and the yaw of the errors in the head pointing error. Pitch direction errors: Target Elevation: $F=16.14 \mathrm{df}=4,5 \mathrm{p}<.009$; Target Azimuth: $\mathrm{F}=7.08, \mathrm{df}=4,5 \mathrm{p}<.027$; Yaw direction errors: Target Elevation: ns; Target Azimuth: $F=29.5 \mathrm{df}=4,32 \mathrm{p}<.001$. Standard errors for the mean error ranged between 1 and 10 degrees. The main effect of the presence of the light cursor was not significant and did not interact with other independent variables (See figs. 3 and 4 ) 
Since we did not anticipate errors as large as actually measured, we did not use spherical statistics to correct the problems of mapping spherical data into a linear analysis. Because the analysis was conducted on the error data corrected for wrap-around of the scale and most of the errors were less than 15 degrees, use of spherical statistics is not likely to substantially change the major results.

The proper method to use to correct for movement of the subject from his calibrated reference position while he positions the cursor depends upon his interpretation of the of the meaning of the cursor position. If he considers its image on the inner surface of the sphere to represent the location of a target cube at that distance, about $1.5 \mathrm{~m}$, he would have to introduce parallax corrections to his body-referenced, head direction as he translated with respect to the original reference point so as to keep the cursor on the same place on the sphere as he moved. Alternatively, if he considered, as he in fact was instructed, the cursor image to represent a body-referenced direction toward the target, head displacement in itself would not require adjustment of head direction to keep to cursor properly pointed. This condition is particularly true since he was instructed that the target was at a relatively great distance from the reference cube. For the layouts used, the distance between target and reference was $6 \mathrm{~m}$ and the viewing distance was modeled at $28 \mathrm{~m}$. At this distance the parallax correction for a $5 \mathrm{~cm}$ lateral movement would have to be only about $0.5^{\circ}$, comparable to the biological noise associated with head direction. Thus, since the head-angle was measured with respect to a bodyreferenced straight ahead, correction for head displacement need not be made.

The observed mean body-referenced errors for both experiments are plotted in figures 3 and 4 as error arcs on a rectangular projection of the response sphere. The pattern shows a tendency to err towards the subject's egocentric straight ahead, but with a significant asymmetry. The results may be interpreted as a composition of errors: 1) the asymmetrical pattern previously reported for exocentric dial responses which is generally away from the straight ahead and 2) a larger but symmetric tendency to overestimate the extent of the gaze direction indicated by the head mounted cursor. Over estimates like this have been reported by Biguer et al. (1984) for hand pointing to visual target and for head pointing to brief auditory targets (Perrott, Ambarsoom, and Tucker, 1987). In the case of hand pointing without visual feedback of pointing error such overestimates result in overshoot errors. In the case of head pointing without pointing error feedback, the overestimates result in undershoot errors similar to those observed.

The observation that the errors were not effected by turning off the light cursor supports the idea that one source of error arises from the proprioceptive and vestibular estimate of head rotation. But whether the phenomena is truly one of gaze remains to be determined by future experiments examining gaze angles produced by different combinations of eye and head angles. The results of the current study clearly show however, the visual direction is a significantly biased metric of virtual space presented by flat panel perspective displays. Modeling and explanation of the causes of the observed biases will allow design of compensated perspective displays. 


\section{REFERENCES}

Barfield, W.; Sandford, J.; and Foley, J.: The mental rotation and perceived realism of computer generated three dimensional images. International Journal of Man-machine studies, 1989, (in press).

Biguer, B.; Prablanc, C.; Jeannerod, M.: The contribution of eye and head movements in hand pointing accuracy. Experimental Brain Research, vol. 55, 1984, pp. 462-469.

Grunwald, Arthur; and Ellis, Stephen R.: Spatial orientation by familiarity cues. Proceedings of the 6th European Annual Conference on Manual Control, June 1986, University of Keele, Great Britain.

Ellis, Stephen R.; Smith, Stephen; McGreevy, Michael W.: Distortions of perceived visual directions out of pictures. Perception and Psychophysics, vol. 42, 1987, pp. 535-544.

Mardia, K. V.: The statistics of directional data Academic Press, New York, 1972.

McGreevy, Michael W.; and Ellis, Stephen R.: The effect of perspective geometry on judged direction. Human Factors, vol. 28, 1986, pp. 439-456.

Perrott, David: Changes in head position as a measure of auditory localization performance: auditory psychomotor coordination under monaural and binaural listening conditions. Journal of the Acoustical Society of America, vol. 82, 1987, pp. 1637-1645.

Rosinski, R. R.; Mulholland, T.; Degelman, D.; and Faber, J.: Picture perception: an analysis of compensation. Perception and Psychophysics, vol. 28, 1980, pp. 512-525.

Sedgwick, H. A.: Space perception in Handbook of Perception and Human Performance vol. 1., K. R. Boff, L. Kaufman, J. P. Thomas eds, New York: Wiley, vol. 21, no. 19-21, 1986. 

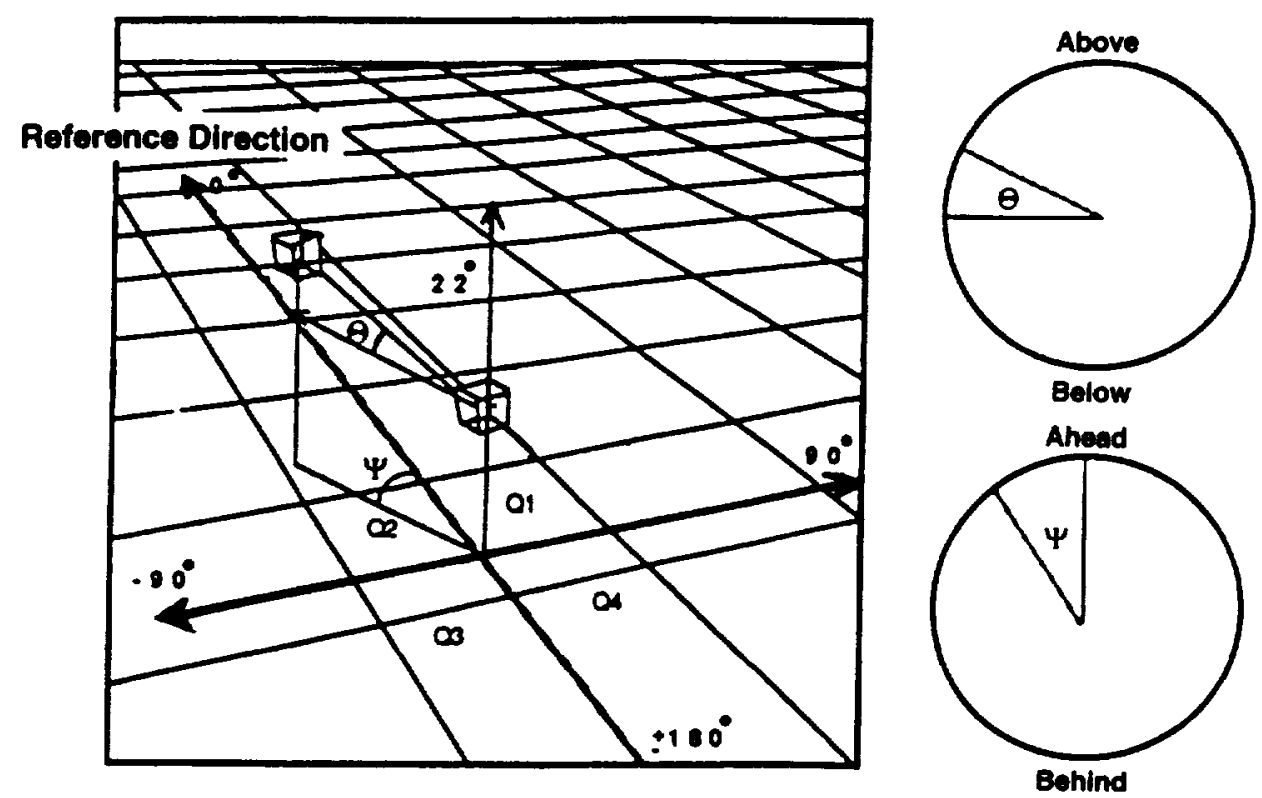

Figure 1. A schematic illustration of the direction judgement task. The subject adjusted the angles $\Psi$ and $\Theta$ shown on the dials at the right until they appeared equal to the azimuth angle $\Psi$ and the elevation angle $\Theta$ of the target cube relative to reference at the center. Dotted lines, labels and arrows did not appear on the map display.
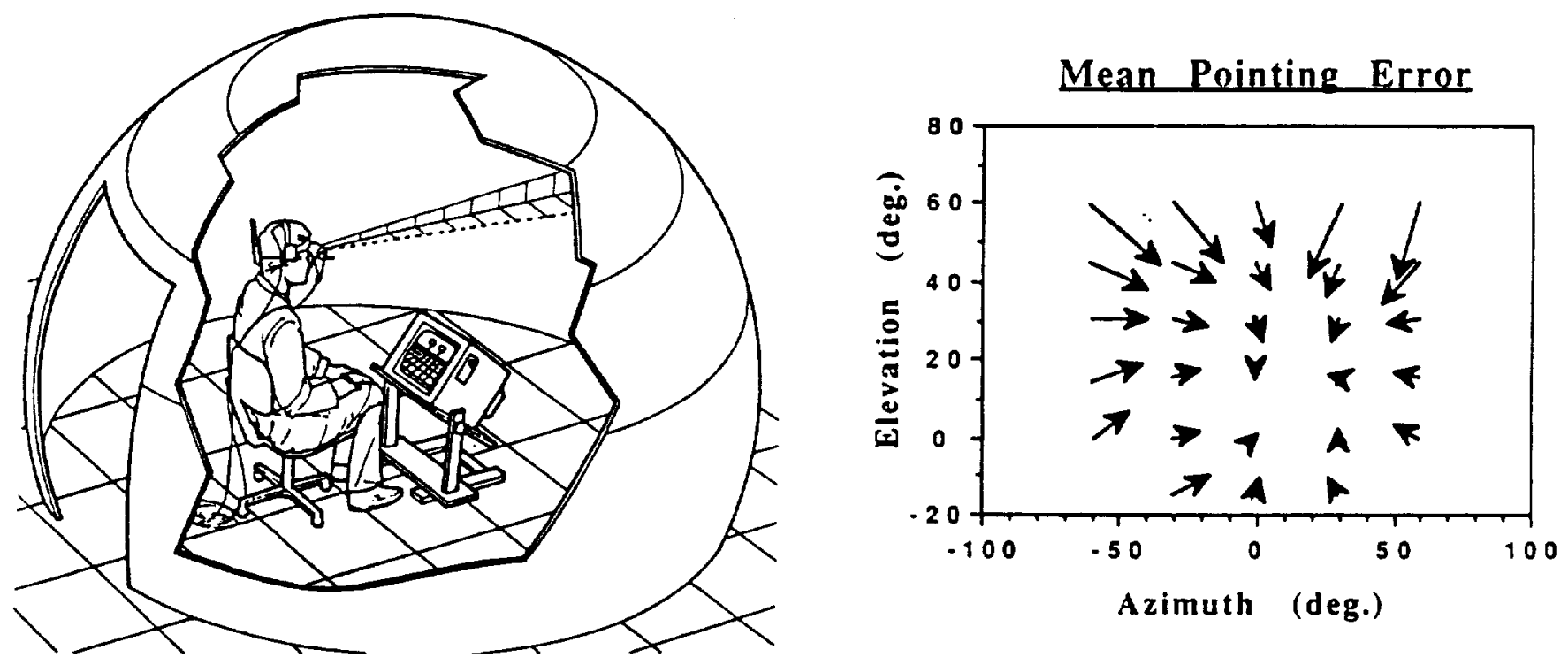

Figure 2. A schematic illustration of the experimental arrangement by which the subject indicated the visual direction at which he would expect to see the target presented on the CRT perspective display if he were positioned at the reference point and aligned with the reference direction. The data in the right portion of the figure represent the average error arcs in a rectangular projection of the forward sphere when both experimental conditions are combined. Each arrow represented the average pitch and yaw error in visual direction to a point at the tail of the arrow. 


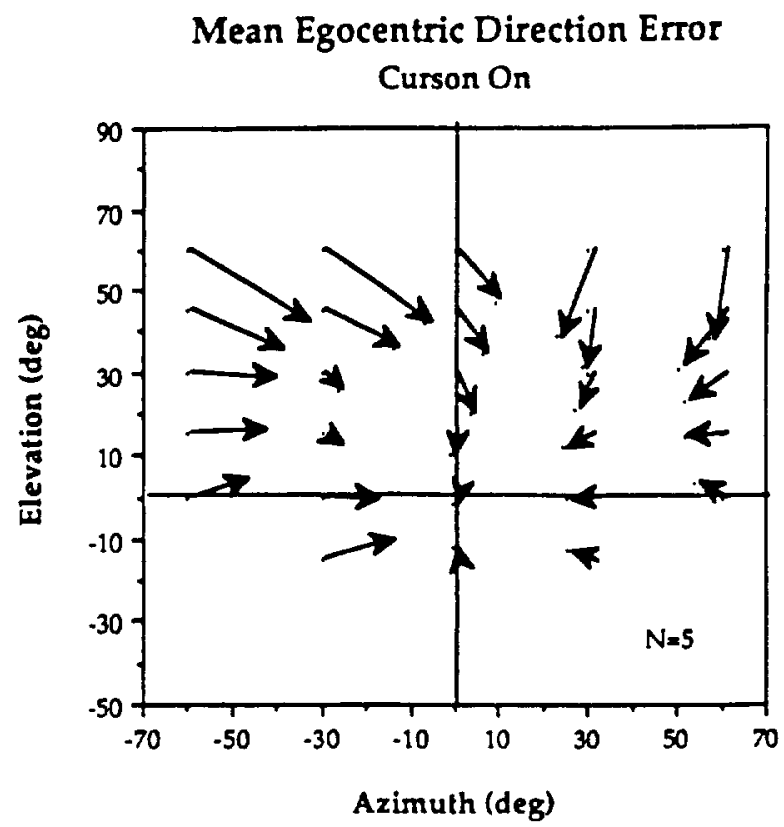

Figure 3. The data in the figure represent the average error arcs in a rectangular projection of the forward sphere for the condition in which the head driven cursor was turned on. Each arrow represents the average pitch and yaw error in visual direction to a point at the tail of the arrow.

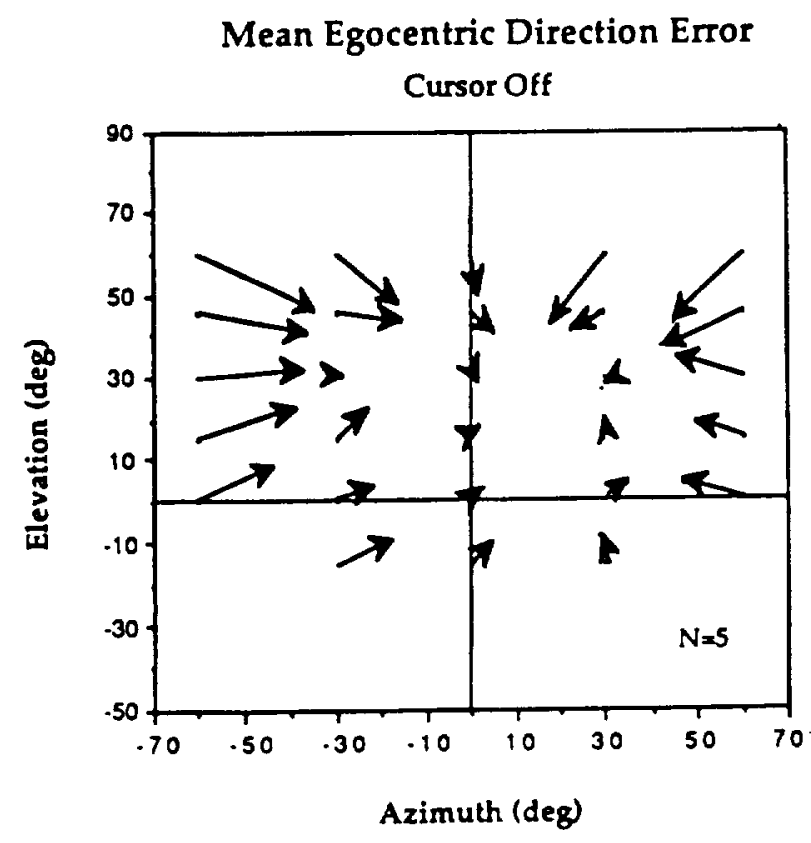

Figure 4. The data in the figure represent the average error arcs in a rectangular projection of the forward sphere for the condition in which the head driven cursor was turned off. Each arrow represents the average pitch and yaw error in visual direction to a point at the tail of the arrow. 


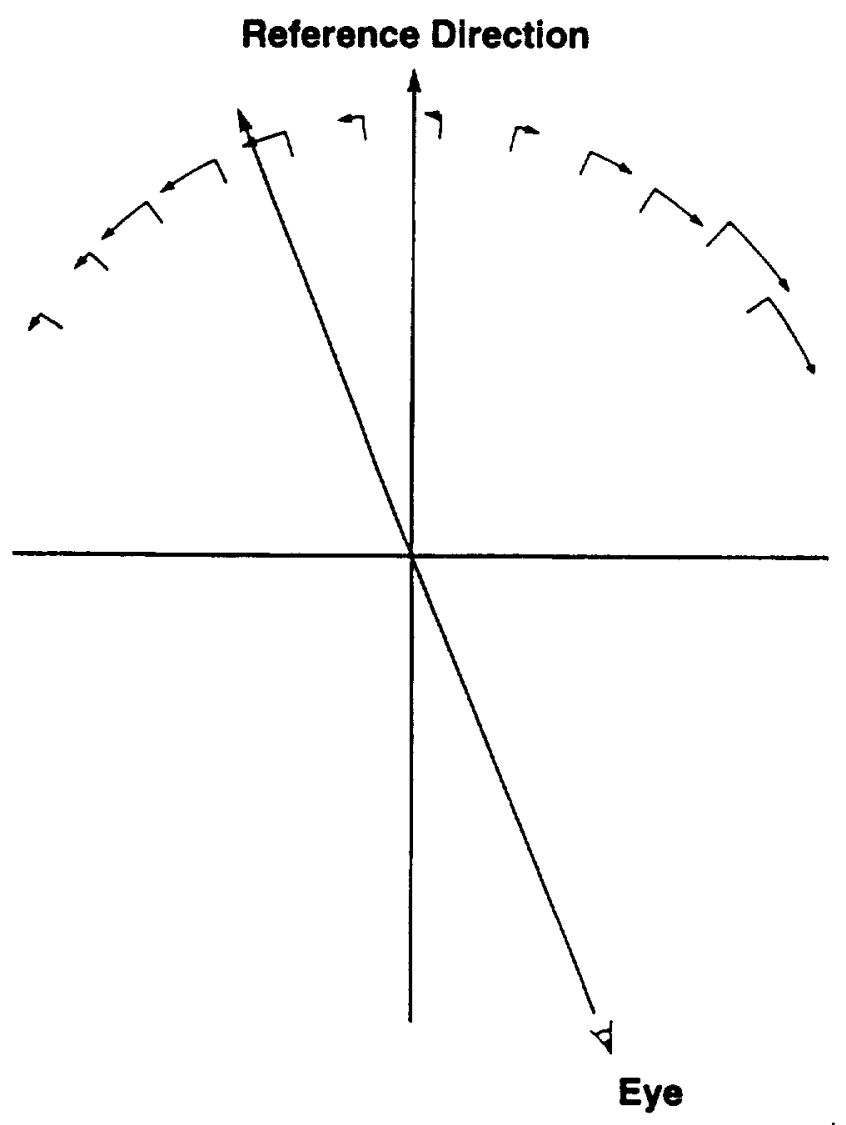

Figure 5. Circular plots for perspective displays in which subjects indicated target azimuth for targets at 0 degrees elevation by adjusting an angle on a dial. The errors are plotted as directed arcs with the tail of each arrow at the correct position of the target. The length of each arrow represents the average error from 8 subjects. Though the viewing azimuth was $-22^{\circ}$ compared to the $-35^{\circ}$ used in the current experiments, the conditions are otherwise comparable. The error arcs clearly show a bias away from the straight ahead rather than towards it and also show an asymmetry with greater errors in the right quadrant than in the left. Thus, if this bias were to cancel a larger one, perhaps due to overestimation of gaze direction, that was toward the straight ahead, the resulting bias would be smaller in the right quadrant than in the left. This expected pattern in found the the data for zero degree target orientation in figures 3 and 4. 